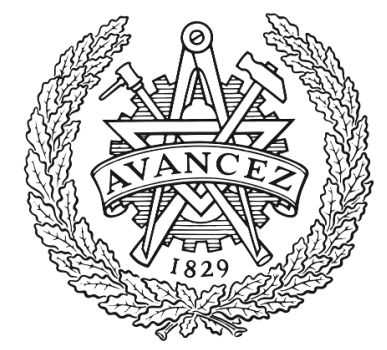

CHALMERS

UNIVERSITY OF TECHNOLOGY

\title{
Electrically pumped graphene-based Landau-level laser
}

Downloaded from: https://research.chalmers.se, 2023-04-26 00:47 UTC

Citation for the original published paper (version of record):

Brem, S., Wendler, F., Winnerl, S. et al (2018). Electrically pumped graphene-based Landau-level laser. Physical Review Materials, 2(3). http://dx.doi.org/10.1103/PhysRevMaterials.2.034002

N.B. When citing this work, cite the original published paper. 


\title{
Electrically pumped graphene-based Landau-level laser
}

\author{
Samuel Brem, ${ }^{1, *}$ Florian Wendler, ${ }^{1}$ Stephan Winnerl, ${ }^{2}$ and Ermin Malic ${ }^{1}$ \\ ${ }^{1}$ Department of Physics, Chalmers University of Technology, 41296 Gothenburg, Sweden \\ ${ }^{2}$ Helmholtz-Zentrum Dresden-Rossendorf, 01314 Dresden, Germany
}

(Received 12 December 2017; published 14 March 2018)

\begin{abstract}
Graphene exhibits a nonequidistant Landau quantization with tunable Landau-level (LL) transitions in the technologically desired terahertz spectral range. Here, we present a strategy for an electrically driven terahertz laser based on Landau-quantized graphene as the gain medium. Performing microscopic modeling of the coupled electron, phonon, and photon dynamics in such a laser, we reveal that an inter-LL population inversion can be achieved resulting in the emission of coherent terahertz radiation. The presented paper provides a concrete recipe for the experimental realization of tunable graphene-based terahertz laser systems.
\end{abstract}

DOI: 10.1103/PhysRevMaterials.2.034002

\section{INTRODUCTION}

Graphene as a two-dimensional material with a linear band structure exhibits a unique Landau quantization in strong magnetic fields [1-5]. The continuous density of states (DOS) within the Dirac cone rearranges into discrete nonequidistant Landau levels (LLs), whose energetic positions can be tuned by changing the magnetic field. Due to the relativistic properties of low-energy electrons in graphene, the spacing between its lowest LLs is extremely large compared to conventional materials. Magnetic fields of a few Tesla give rise to LL transition energies ranging from the terahertz $(\mathrm{THz})$ up to the far-infrared spectral ranges, which makes Landau-quantized graphene a highly promising material for the realization of tunable THz emitters and absorbers [6-10].

In previous work [10] we have shown that the remarkable properties of Landau-quantized graphene can be exploited to achieve continuous-wave lasing with an optically pumped population inversion (PI) between neighboring LLs. However, the optical excitation is highly challenging since one has to selectively address single LLs with energetic separations of a few tens of meV. Therefore, an electrically driven LL laser would be technologically favorable. Here, our current paper sets in and predicts an electrical pump scheme to realize tunable terahertz lasers based on Landau-quantized graphene. Here, the PI between LLs is achieved via selective carrier injection by utilizing highly doped semiconductor leads. To microscopically investigate the proposed mechanism and to reveal the experimental conditions needed for such an electrically driven LL laser, we use a quantum-mechanical approach to describe the coupled dynamics of electrons, phonons, and photons in the laser system. Based on our model, we provide profound insight into the complex interplay of pumping, stimulated, and spontaneous emission of photons as well as Coulomb- and phonon-induced nonradiative processes allowing us to predict optimal conditions for lasing at tunable frequencies.

\footnotetext{
*samuel.brem@chalmers.se
}

\section{LASING MECHANISM}

We propose to embed the graphene layer between differently doped semiconductor leads, giving rise to a double heterostructure similar to those which are used for lead-salt lasers [11,12]. Figure 1 schematically illustrates the lasing scheme consisting of an $n$-doped semiconductor (the left side), the graphene layer (the middle), and a $p$-doped semiconductor (the right side). The entire system is penetrated by a magneticfield $B$, which induces discrete LLs $l$ in graphene [with energy $\left.\varepsilon_{l} \propto \operatorname{sgn}(l) \sqrt{B|l|}\right]$, whereas the density of states within the semiconductors stays quasicontinuous due to large effective electron masses. Moreover, for a good performance of the laser it is necessary that the Fermi energy in graphene is close to the Dirac point in graphene so that it is pinned in the zeroth LL when a magnetic field is applied. Additionally, the semiconductors should be doped symmetrically, i.e., in the biased structure the conduction-band minimum of the $n$-doped lead $E_{\mathrm{n}}$ has the same distance to the Fermi energy in the graphene layer as the valance-band maximum $E_{\mathrm{p}}$ of the $p$-type semiconductor. Note that we neglect the valence (conduction) band of the $n$-type ( $p$-type) semiconductor since it is assumed to lie energetically much lower (higher) than the LLs involved in the laser scheme.

By applying a bias voltage $U_{L}$ in the forward direction of the $p$ - $n$ junction (cf. the red horizontal arrow in Fig. 1), we induce a strong nonequilibrium carrier distribution throughout the heterostructure exhibiting different quasi-Fermi levels for electrons and holes within the $n$ - and $p$-type semiconductors, respectively. Therewith we obtain a spectrally narrow electron reservoir in the left lead, allowing to selectively inject electrons into resonant LLs within the red shaded channel in Fig. 1. The same principle simultaneously injects holes in the valence band of graphene. Thereby the energetic positions and the widths of the injection channels are determined by the position of the conduction- (valance-) band minimum (maximum) in the left (right) lead and the applied electrical potential. By changing the magnetic field we can now adjust the energetic configuration of LLs in graphene and therewith choose which levels are pumped. In a certain magnetic-field range we can achieve the situation displayed in Fig. 1 where the first LL 


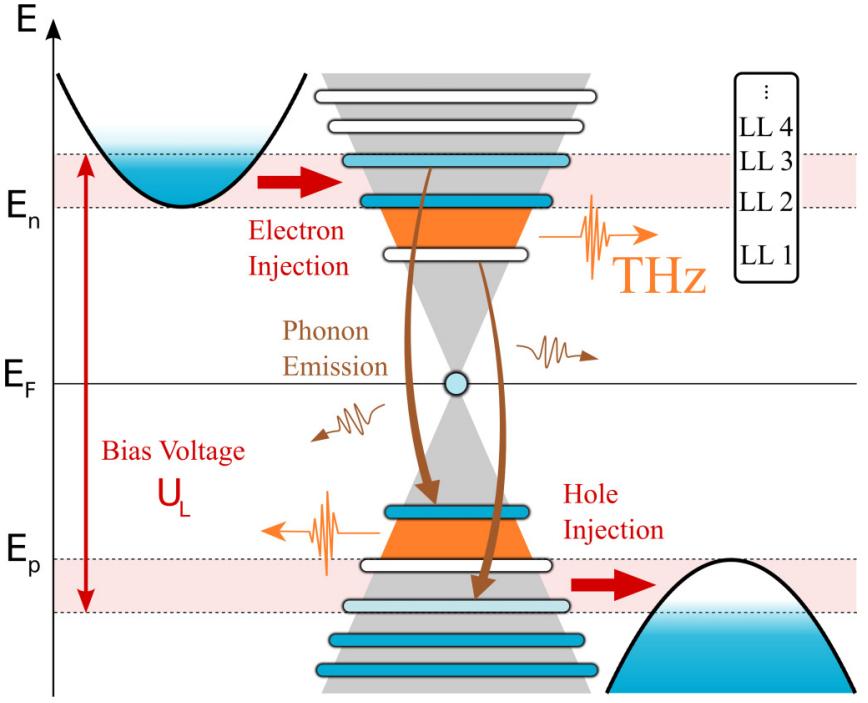

FIG. 1. Scheme of the electrically pumped Landau-level laser. The Landau-quantized graphene (middle) is embedded between an $n$ doped (left) and a $p$-doped semiconductor (right) allowing a selective injection of electrons and holes into resonant LLs within the red shaded channels. This results in a population inversion between LL 1 and LL 2 in the conduction band and their counterparts in the valence band.

$l=+1$ lies below the band minimum of the left lead, whereas the second $l=+2$ and the third $l=+3$ are pumped since they overlap with the injection channel. Hence, we can electrically drive a population inversion between the first two LLs (orange shaded transition). The same holds for $l=-1$ and $l=-2$ in the valence band of graphene due to the selective injection of holes.

To achieve stimulated emission, the system is embedded into a high-quality microcavity $[13,14]$ with a resonator mode matching the energy difference between $l=+1$ and $l=+2$. Therewith the cavity photons become multiplied in an avalanche of stimulated transitions $2 \rightarrow 1$ or $-1 \rightarrow-2$ creating coherent $\mathrm{THz}$ light, cf. the orange arrows in Fig. 1. For continuous lasing, the emission of cavity photons has to be complemented by a nonradiative carrier relaxation to quickly depopulate (repopulate) the lower (upper) laser level $l=+1$ $(l=-2)$. In our proposed pump scheme this is achieved by additionally injecting electrons (holes) in $l=+3(l=-3)$. By choosing the right magnetic field we can achieve resonance between one of the optical phonon modes of graphene and the LL transition $1 \rightarrow-3$ yielding a fast evacuation of carriers in the lower laser level $[10,15]$ as denoted with the brown arrow in Fig. 1. Note that phonon-induced decay rates strongly depend on how well the transitions match the phonon energy so that the efficiency of the laser scheme varies with the magnetic field.

\section{MODELING OF LASER DYNAMICS}

To model the described laser system we apply a manyparticle approach including carrier-carrier, carrier-phonon, and carrier-photon interactions on a microscopic level [16-20], resulting in the following set of coupled equations of motions for carrier occupation $\rho_{l}(t)$ of the $l$ th LL and the photons' number $n_{\mu}(t)$ within the cavity mode $\mu$,

$$
\begin{gathered}
\dot{\rho}_{l}=\sum_{\mu l^{\prime}}\left(S_{l^{\prime} l}^{\mu}-S_{l l^{\prime}}^{\mu}\right)+P_{l}\left(\rho_{l}\right)+\sum_{l^{\prime}} \Gamma_{l l^{\prime}}\left(\rho_{l}, \rho_{l^{\prime}}\right), \\
\dot{n}_{\mu}=4 N_{B} \sum_{l l^{\prime}} S_{l l^{\prime}}^{\mu}-\kappa_{\mu} n_{\mu} .
\end{gathered}
$$

Here $S_{l l^{\prime}}^{\mu}$ denotes the probability of a LL transition $l \longrightarrow l^{\prime}$ via emission or absorption of a photon in mode $\mu$, which is dynamically calculated within a quantum-optical approach. Here we include carrier-photon correlations up to the quadruplet level allowing us to calculate the second-order correlation function of the emitted light $[21,22]$. We consider a finite photon lifetime $\left(\kappa_{\mu}\right)^{-1}=Q / \omega_{\mu}$ that is given by the cavity quality factor $Q$ and the photon frequency $\omega_{\mu}$. Moreover, the rates $\Gamma_{l l}$ incorporate all electron-electron and electron-phonon scattering channels, i.e., all energy allowed LL transitions, including time-dependent Pauli blocking terms. These scattering terms lead to a redistribution of carriers along all LLs towards a Fermi distribution. The explicit equations for $S_{l l^{\prime}}^{\mu}$ and $\Gamma_{l l^{\prime}}$ including all matrix elements can be found in Ref. [10].

In this paper, we focus on the mechanism of electrical pumping. The tunneling of carriers between the leads and the $l$ th $\mathrm{LL}$ is modeled via

$$
P_{l}=P_{0} \sum_{\lambda} \int d \varepsilon\left[f_{\lambda}(\varepsilon)-\rho_{l}\right] \mathcal{L}_{\gamma_{l}}\left(\varepsilon_{l}-\varepsilon\right) \theta_{\lambda}(\varepsilon) .
$$

Here the leads $\lambda=n, p$ are treated within the bath approximation so that the carrier reservoir follows a static Fermidistribution $f_{\lambda}(\varepsilon)=\left\{\exp \left[\left(\varepsilon-\Phi_{\lambda}\right) /\left(k_{B} T\right)\right]+1\right\}^{-1}$, whose quasi-Fermi level is given by the applied electrical potential $\Phi_{\lambda}= \pm U_{L} / 2$. Apart from the occupation difference, the pump rate is further determined by the spectral overlap of the DOS of the leads and the involved LLs that is given by a Lorentzian $\mathcal{L}_{\gamma_{l}}$ with the width $\gamma_{l}$. The leads are assumed to be quasitwo dimensional with a constant DOS reflected by the heavy-side function $\theta_{\lambda}$, whose onset is located at the corresponding band extrema $E_{\lambda}$. The widths of the LLs are calculated dynamically based on the scattering rates $\Gamma_{l l^{\prime}}[10,23-25]$.

To derive Eq. (3) we have assumed that the tunneling matrix element for transitions between electronic states in the lead and LL orbits is constant. Together with the absolute DOS within the leads this parameter determines the pump efficiency $P_{0}$. We can relate this material parameter to the injected current density when neglecting Pauli blocking (e.g., lead is fully occupied, and LLs are empty) and assuming that a certain number $N_{\mathrm{ch}}$ of LLs is fully covered by the injection channel. In that case the current density injected from lead $\lambda$ is given by

$$
j^{\lambda}=\frac{e_{0}}{A} \sum_{l} P_{l}^{\lambda} \approx 4 \frac{e}{A} N_{\mathrm{ch}} N_{B} P_{0}=N_{\mathrm{ch}} \frac{B}{\Phi_{0}} I_{0} .
$$

Here $N_{B}=B A / \Phi_{0}$ denotes the magnetic-field-dependent LL degeneracy reflecting the number of magnetic flux quanta $\Phi_{0}=h / e_{0}$ within the graphene layer of area $A$. The factor 4 further accounts for the spin and valley degeneracies. In the last expression of Eq. (4) we have defined the maximum current per LL as $I_{0}=4 e P_{0}$, which multiplied with the density of magnetic flux quanta (LL degeneracy) gives an estimate of the injected current density per LL. Therefore, we will in 
the following use $I_{0}$ as the central experimentally accessible parameter characterizing the injection efficiency.

\section{LASER CHARACTERISTICS}

To reveal under which conditions the proposed laser mechanism works, we numerically solve the above-described equations for different system parameters. In the following we present the laser dynamics for an optimal parameter configuration assuming a high cavity quality factor of $Q=$ 5000 , a temperature of $T=300 \mathrm{~K}$, a laser cross section of $A=1000 \mu \mathrm{m}^{2}$, and an efficient carrier injection determined by $I_{0}=2 \mu \mathrm{A}$. The photon lifetime (given by $Q$ ) strongly depends on the design of the utilized cavity and significantly influences the threshold condition of a laser. Since our paper focuses on many-particle processes and intrinsic loss channels of the laser active medium, we have chosen a high cavity quality to make our paper independent of the cavity design. However, first experimental studies performed on $\mathrm{THz}$ cavities have demonstrated very large photon lifetimes corresponding to quality factors of several thousands [26]. Therefore, we think that a variety of high- $Q$ cavity designs comparable with current optical-range cavities [27] will be available in the near future, including designs compatible with the proposed heterostructure. Moreover, we choose a magnetic field of $B=2.7 \mathrm{~T}$ to reach a resonance between the LL transition $1 \rightarrow-3$ and transverse optical K-phonons (KTO) $\left(\varepsilon_{\text {KTO }}=\right.$ $162 \mathrm{meV}[28,29])$. Now we choose the position and width of the injection channel according to the situation displayed in Fig. 1. Hence we set $E_{F}=0, E_{n}=-E_{p}=\varepsilon_{l=2}(B=$ $2.7 \mathrm{~T}) \approx 84 \mathrm{meV}$ and $U_{L}=2 \Phi_{c} / e=2 \varepsilon_{l=3}(B=2.7 \mathrm{~T}) / e \approx$ $205 \mathrm{mV}$. Now only half of the second and third LLs are covered by the injection channel, which reduces the pump efficiency of those two levels. However we want to avoid any overlap of the first and fourth LLs with the injection channel since this would strongly counteract the PI.

Figure 2 shows the dynamics of the electrically pumped LL laser, characterized by the population difference $\rho_{2}-\rho_{1}$ [the black curve in Fig. 2(a)], the total number of photons in the cavity (the orange curve), and the second-order autocorrelation function $g^{(2)}$ of the cavity light [Fig. 2(b)]. In the beginning $(t=0)$ the system is set to thermal equilibrium, reflected by a Fermi distribution for LLs $(\mathrm{PI}<0)$ and very few photons. At 50 ps we slowly increase the bias voltage which reaches its maximum at $100 \mathrm{ps}$. Instead of an instantaneous switch on, we choose a smooth step function for the voltage with a Gaussian slope. As soon as the voltage is turned on, the amount of electrons in the $n$-doped lead rapidly increases, which enhances the tunneling efficiency giving rise to an increased occupation of the second LL $\rho_{2}$. Already after 75 ps a population inversion is reached, i.e., $\rho_{2}-\rho_{1}>0$. The PI increases adiabatically with the voltage up to a quasistationary PI value of about $23 \%$ at 100 ps. Here, the injection of carriers and phonon-induced relaxation into lower LLs are in balance yielding stable occupations.

Simultaneously, the number of photons increases exponentially via stimulated emission once a PI is established (cf. the orange curve). For about $50 \mathrm{ps}$ the PI stays stable despite the growing photon avalanche. However, after approximately 150 ps the PI slowly decreases reflecting the so-called gain

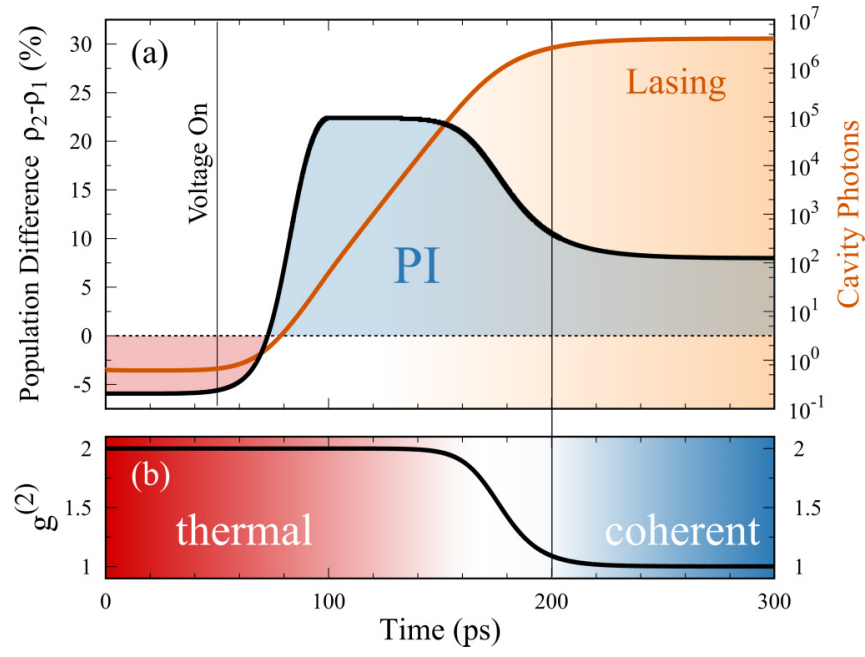

FIG. 2. Time evolution of (a) the population difference $\rho_{2}-\rho_{1}$ (the black curve) and the total number of photons in the cavity (the orange curve) and (b) the second-order correlation function $g^{(2)}$ of the cavity light. Once a voltage is switched on, a population inversion, i.e., $\rho_{2}-\rho_{1}>0$, can be reached resulting in an exponential increase in the photon number and $g^{(2)} \rightarrow 1$ characterizing coherent laser light.

compression [10], and the number of photons saturates. At about 200 ps the system reaches a new equilibrium of pumping, photon emission, and nonradiative relaxation characterizing the lasing regime. Finally, we also have access to the photon statistics of the emitted light via the second-order correlation function $g^{(2)}$, cf. Fig. 2(b). Before the voltage is switched on the photons are completely uncorrelated, which is reflected by $g^{(2)}=2$ characterizing thermal light. Once a population inversion is reached, the number of photons increases due to stimulated emissions, and $g^{(2)}$ approaches the value of 1 characterizing coherent laser light.

The dynamics presented in Fig. 2 shows that the proposed lasing scheme works and leads to the emission of coherent $\mathrm{THz}$ radiation for a certain set of parameters. In the next step we widely vary the experimental conditions to determine the minimum injection efficiency (threshold current) and how tunable the laser will be for a fixed lead configuration. Here we keep the cavity quality and position of the injection channel constant and subsequently vary the injection efficiency $I_{0}$ and the magnetic-field $B$. Figure 3 shows the laser output power in equilibrium in dependence of: (a) the injection efficiency $I_{0}$ (for a fixed $B=2.7 \mathrm{~T}$ ) and (b) the magnetic field (for a fixed $\left.I_{0}=2 \mu \mathrm{A}\right)$. We find a strong increase in the output power at a threshold value of about $I_{0}^{\text {th }}=0.3 \mu \mathrm{A}$ [the dashed line in Fig. 3(a)]. For injection efficiencies below this value, we obtain a very low photon output due to the predominantly spontaneous emission. In this parameter region the injection rate is too slow compared to phonon relaxation processes in the graphene sheet so that the resulting PI does not induce sufficient optical gain to compensate the cavity losses. The underlying microscopic mechanisms limiting the efficiency of an optically pumped LL laser have been already discussed in Ref. [10]. Here, in the electrically pumped scenario, we find that, for the above-mentioned setup, the injection efficiency has to be on the order of $I_{0}^{\text {th }}=0.3 \mu \mathrm{A}$ to achieve lasing. 

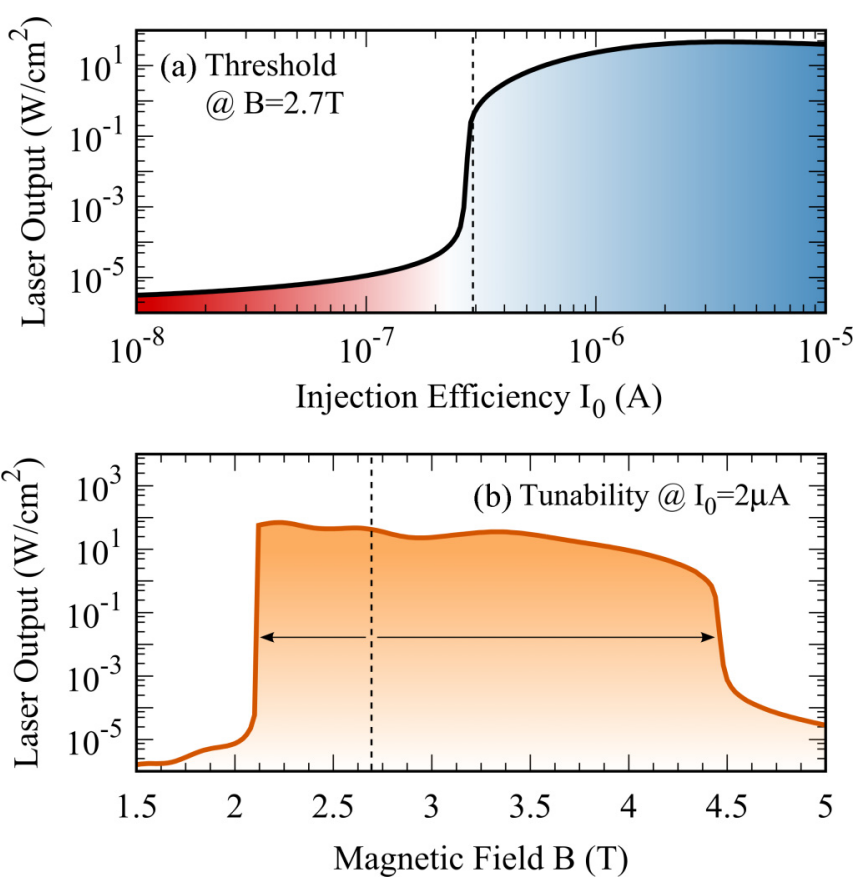

FIG. 3. Laser output power in equilibrium as function of (a) the injection efficiency $I_{0}$ (for a fixed $B=2.7 \mathrm{~T}$ ) and (b) the magneticfield $B$ (for a fixed $I_{0}=2 \mu \mathrm{A}$ ). Whereas (a) reveals a threshold current of $I_{0}^{\text {th }}=0.3 \mu \mathrm{A}$, (b) illustrates the wide tunability of the laser mechanism.

Applying Eq. (4) we can estimate the injected current per LL by multiplying this current with the density of magnetic flux quanta, yielding a threshold current density of about $40 \mathrm{kA} / \mathrm{cm}^{2}$. Note that this is a rough estimate since the effect of Pauli blocking has not been taken into account. The dynamical calculation of the current including the occupation differences between the lead and the LL yields a threshold current of about $12 \mathrm{kA} / \mathrm{cm}^{2}$. Whereas metallic leads form Ohmic contacts on graphene with very low contact resistance [30], graphenesemiconductor contacts are Schottky type [31,32]. However, there are so far - to the best of our knowledge - no experimental studies in literature reporting on graphene embedded into a $p-n$ junction. Therefore, a direct evaluation of the feasibility of the calculated threshold value is not possible. In the proposed device the current will flow in the forward direction through the $p-n$ junction of the low gap semiconductor. As shown from lead-salt lasers, such devices enable current densities in the range of $10 \mathrm{kA} / \mathrm{cm}^{2}$ [11], which shows that the required current densities are, in principle, feasible. Nevertheless, the realization of the proposed heterostructure with good contacts to the semiconducting materials and the combination with a high cavity quality will be challenging and require considerable technological effort.
In Fig. 3(b), we now vary the magnetic field to examine how tunable the proposed electrically driven LL laser can be. The injection efficiency stays constant at the exemplary value of $I_{0}=2 \mu \mathrm{A}$, and the position as well as the width of the injection channel are fixed to the same configuration as before. Hence, when changing the magnetic field, the pump conditions drastically change since the LLs, which are supposed to be pumped, move out (whereas other LLs move in) the injection channel. However, Fig. 3(b) shows that the laser scheme works in a wide range of magnetic fields with the same lead configuration, achieving output powers above $10 \mathrm{~W} / \mathrm{cm}^{2}$ between about $B_{\min }=2.1$ and $B_{\max }=4.5 \mathrm{~T}$. In consequence, the proposed LL laser works in a range between 5.3 and $7.7 \mathrm{THz}$.

For magnetic fields lower than the optimum value of $B=2.7 \mathrm{~T}$ (the dashed line) we achieve the highest output powers. Here the upper laser level $l=2$ shifts below the band edge of the $n$-doped lead so that a PI between $l=3$ and $l=2$ is driven. Although the transition energy of $3 \rightarrow 2$ is detuned from the transition $2 \rightarrow 1$ (cavity resonance), we still obtain stimulated emission into the cavity mode due to the strong dephasing induced by phonon and Coulomb scatterings. Subsequently, carriers accumulating in $l=2$ after the emission via the $3 \rightarrow 2$ lead to an inversion between $l=2$ and $l=1$ allowing for a second laser transition $2 \rightarrow 1$. This cascade of laser transitions allows a very efficient laser operation and extends the magnetic-field range down to $B_{\min }=2.1 \mathrm{~T}$ where also the third LL shifts below the injection channel. For high magnetic fields the tunability is limited by the pumping of the lower laser level $l=1$, which starts to enter the injection channel at about $B_{\max }=4.5 \mathrm{~T}$.

In conclusion, we present a mechanism for electrical pumping of a graphene-based terahertz Landau-level laser. To provide a concrete recipe for the experimental realization of such lasers, we perform a fully quantum-mechanical investigation of the nonequilibrium dynamics of carriers, phonons, and photons in such a laser. Our calculations reveal that, in order to achieve the laser threshold conditions, cavities with $Q$ factors of several thousands and current densities between the graphene and the doped semiconductor in the range of $\mathrm{kA} / \mathrm{cm}^{2}$ are required. Under these conditions the proposed Landau-level laser emits coherent terahertz radiation with high output power and can be externally tuned within a continuous range between 5.3 and $7.7 \mathrm{THz}$.

\section{ACKNOWLEDGMENTS}

This project has received funding from the European Union's Horizon 2020 Research and Innovation Programme under Grant Agreement No. 696656. Furthermore, we acknowledge financial support from the Swedish Research Council (VR).
[1] K. S. Novoselov et al., Two-dimensional gas of massless dirac fermions in graphene, Nature (London) 438, 197 (2005).

[2] D. L. Miller et al., Observing the quantization of zero mass carriers in graphene, Science 324, 924 (2009).
[3] M. O. Goerbig, Electronic properties of graphene in a strong magnetic field, Rev. Mod. Phys. 83, 1193 (2011).

[4] M. L. Sadowski, G. Martinez, M. Potemski, C. Berger, and W. A. de Heer, Landau Level Spectroscopy of Ul- 
trathin Graphite Layers, Phys. Rev. Lett. 97, 266405 (2006).

[5] F. Wendler, A. Knorr, and E. Malic, Ultrafast carrier dynamics in Landau-quantized graphene, Nanophotonics 4, 224 (2015).

[6] H. Aoki, Novel Landau level laser in the quantum Hall regime, Appl. Phys. Lett. 48, 559 (1986).

[7] T. Morimoto, Y. Hatsugai, and H. Aoki, Cyclotron radiation and emission in graphene, Phys. Rev. B 78, 073406 (2008).

[8] F. Wendler and E. Malic, Towards a tunable graphene-based Landau level laser in the terahertz regime, Sci. Rep. 512646 (2015).

[9] Y. Wang, M. Tokman, and A. Belyanin, Continuous-wave lasing between Landau levels in graphene, Phys. Rev. A 91, 033821 (2015).

[10] S. Brem, F. Wendler, and E. Malic, Microscopic modeling of tunable graphene-based terahertz landau-level lasers, Phys. Rev. B 96, 045427 (2017).

[11] H. Preier, M. Bleicher, W. Riedel, and H. Maier, Double heterojunction pbs-pbs1- $\mathrm{x}$ se $\mathrm{x}$-pbs laser diodes with cw operation up to 96 k, Appl. Phys. Lett. 28, 669 (1976).

[12] M. Tacke, New developments and applications of tunable ir lead salt lasers, Infrared Phys. Technol. 36, 447 (1995).

[13] M. Engel et al., Light-matter interaction in a microcavitycontrolled graphene transistor, Nat. Commun. 3, 906 (2012).

[14] G. Wysocki et al., Widely tunable mode-hop free external cavity quantum cascade laser for high resolution spectroscopic applications, Appl. Phys. B 81, 769 (2005).

[15] F. Wendler, A. Knorr, and E. Malic, Resonant carrier-phonon scattering in graphene under landau quantization, Appl. Phys. Lett. 103, 253117 (2013).

[16] H. Haug and S. W. Koch, Quantum Theory of the Optical and Electronic Properties of Semiconductors (World Scientific, Singapore, 1990), Vol. 5.

[17] F. Rossi, and T. Kuhn, Theory of ultrafast phenomena in photoexcited semiconductors, Rev. Mod. Phys. 74, 895 (2002).

[18] M. Kira and S. Koch, Many-body correlations and excitonic effects in semiconductor spectroscopy, Prog. Quantum Electron. 30, 155 (2006).
[19] E. Malic and A. Knorr, Graphene and Carbon Nanotubes: Ultrafast Optics and Relaxation Dynamics (Wiley, Hoboken, NJ, 2013).

[20] F. Kadi, T. Winzer, E. Malic, A. Knorr, F. Göttfert, M. Mittendorff, S. Winnerl, and M. Helm, Microscopic Description of Intraband Absorption in Graphene: The Occurrence of Transient Negative Differential Transmission, Phys. Rev. Lett. 113, 035502 (2014).

[21] C. Gies, J. Wiersig, M. Lorke, and F. Jahnke, Semiconductor model for quantum-dot-based microcavity lasers, Phys. Rev. A 75, 013803 (2007).

[22] R. Jago, T. Winzer, A. Knorr, and E. Malic, Graphene as gain medium for broadband lasers, Phys. Rev. B 92, 085407 (2015).

[23] T. Ando and Y. Uemura, Theory of quantum transport in a two-dimensional electron system under magnetic fields. i. characteristics of level broadening and transport under strong fields, J. Phys. Soc. Jpn. 36, 959 (1974).

[24] N. H. Shon and T. Ando, Quantum transport in two-dimensional graphite system, J. Phys. Soc. Jpn. 67, 2421 (1998).

[25] H. Funk, A. Knorr, F. Wendler, and E. Malic, Microscopic view on Landau level broadening mechanisms in graphene, Phys. Rev. B 92, 205428 (2015).

[26] T. Chen, P. Liu, J. Liu, and Z. Hong, A terahertz photonic crystal cavity with high q-factors, Appl. Phys. B 115, 105 (2014).

[27] K. J. Vahala, Optical microcavities, Nature (London) 424, 839 (2003).

[28] S. Piscanec, M. Lazzeri, F. Mauri, A. C. Ferrari, and J. Robertson, Kohn Anomalies and Electron-Phonon Interactions in Graphite, Phys. Rev. Lett. 93, 185503 (2004).

[29] J. Maultzsch, S. Reich, C. Thomsen, H. Requardt, and P. Ordejón, Phonon Dispersion in Graphite, Phys. Rev. Lett. 92, 075501 (2004).

[30] R. Ribeiro-Palau et al., Quantum hall resistance standard in graphene devices under relaxed experimental conditions, Nat. Nanotechnol. 10, 965 (2015).

[31] S. Tongay, M. Lemaitre, X. Miao, B. Gila, B. R. Appleton, and A. F. Hebard, Rectification at Graphene-Semiconductor Interfaces: Zero-Gap Semiconductor-Based Diodes, Phys. Rev. X 2, 011002 (2012).

[32] H. Yang et al., Graphene barristor, a triode device with a gatecontrolled schottky barrier, Science 336, 1140 (2012). 